\title{
ESTUDIOS
}

\section{El uso de la etnografía en el estudio de las músicas mapuche}

\section{The Use of Ethnography in the Study of Mapuche Music}

\author{
por \\ Leonardo Díaz-Collao \\ Universidad de Los Lagos, Chile \\ leodiazcollao@gmail.com \\ Ignacio Soto-Silva \\ Universidad de Los Lagos, Chile \\ ignacio.soto@ulagos.cl
}

\begin{abstract}
El presente artículo surge a propósito de la experiencia etnográfica y la reflexión teórico-metodológica de sus autores en el marco de sus investigaciones doctorales y proyectos asociados. El objetivo de este trabajo es analizar el uso de la etnografía en Chile en el estudio de las músicas mapuche. Para esto realizamos una búsqueda bibliográfica en diversas fuentes de información (repositorios de tesis, bases de datos académicas, espacios de difusión vinculados a organizaciones mapuche) y revisamos los trabajos acerca de expresiones musicales mapuche que utilizan la metodología en cuestión o bien algún "componente" etnográfico en sus enfoques cualitativos. El cuerpo analizado comprende publicaciones desde 1970 hasta principios de 2019. En los treinta y dos textos revisados, identificamos los siguientes aspectos metodológicos: temas de estudio, localización de los trabajos, duración del trabajo de campo y técnicas de recolección de información, para luego reflexionar acerca del modo en que estos comprenden y desarrollan la etnografía entendida como encuadre metodológico y, secundariamente, texto. Por último, reflexionamos acerca de los posibles factores que inciden en el escaso uso de la etnografía en el estudio de las músicas mapuche, metodología que además de ser poco empleada en las investigaciones de las expresiones sonoras de esta sociedad, es, en muchos casos, utilizada de manera parcial.

Palabras clave: Revisión bibliográfica, etnografía, música mapuche, investigación cualitativa.
\end{abstract}

This paper emerges based on the ethnographic experience and theoretical-methodological reflection of its authors in the framework of their doctoral research and research project. The aim of this review is to analyze the use of ethnography in the study of Mapuche music, in Chile. We carry out a bibliographic review in various sources of information (thesis repositories, academic databases, dissemination spaces associated with mapuche organizations) and select works that address mapuche musical expressions that use the methodology in question, or some ethnographic "component" in their qualitative approaches. The analyzed corpus includes publications from 1970 to early 2019. In the thirty-two works reviewed, we identified the following methodological aspects: study topics, location of the work, duration of the field work and data collection techniques. Subsequently, we reflect on how they understand and develop ethnography as a methodological framework and as text. Finally, we reflect on the possible factors that affect the modest use of ethnography in the study of Mapuche music, a methodology that, in addition to being rarely used in the investigations of the sound expressions of this society, is, in many cases, partially used.

Keywords: Literature review, ethnography, Mapuche music, qualitative research. 


\section{ANTECEDENTES 1}

La etnografía es, probablemente, una de las metodologías más empleadas a la hora de estudiar manifestaciones musicales tradicionales o urbanas. Esto no solo se debe a que, en varios casos, estas se caracterizan por su transmisión mediante la tradición oral; sino también por el énfasis disciplinario en la experiencia musical y la relevancia de las condiciones sociales, políticas y culturales en las que se desarrollan. Estas requieren que el investigador o investigadora se desplace e involucre por un tiempo significativo con el grupo o comunidad con el que trabaja. En el presente artículo hemos revisado el uso de la etnografía en el estudio de las músicas mapuche ${ }^{2}$. Nuestro objetivo es reflexionar acerca de la presencia y frecuencia con la que esta metodología ha sido utilizada en la observación y representación de la cultura mapuche. Buscamos nutrir el debate etnográfico mediante un "objeto" -la música-, que pareciera ser marginal en disciplinas como la antropología; y por otra parte, evaluar la relevancia y conveniencia de un método poco utilizado por la musicología en Chile. Las reflexiones aquí expuestas surgen a propósito de nuestras experiencias en el trabajo de campo y se basan en la revisión bibliográfica de diversas fuentes (libros, capítulos de libros, tesis de posgrado, artículos y otros). Hemos decidido atender a los estudios en torno a la cultura musical mapuche por tres razones: 1) el vínculo entre nuestros proyectos de investigación y esta línea de estudio, 2) la importancia que tiene esta sociedad en Chile y América del Sur, y 3) la necesidad de discutir respecto de los problemas éticos, metodológicos y epistemológicos en un contexto caracterizado por una clara situación colonial.

Los mapuche son el pueblo indígena más numeroso de Chile con 1.745 .147 personas, que representa el 9,9\% del total de la población efectivamente censada en 2017 (INE 2018), y cuentan con una importante presencia en algunas provincias de Argentina. La relevancia de este pueblo se debe, también, a las tensiones actuales entre el Estado chileno y las comunidades mapuche, como resultado de los procesos coloniales que comienzan en el siglo XIX y se proyectan hasta la actualidad. María José Barros (2009) atribuye a los medios de comunicación masiva la responsabilidad en la masificación de la concepción de "conflicto mapuche" y su contribución a la construcción de ciertos estereotipos que han impactado en una visión negativa del indígena, tesis que es apoyada por otros autores ${ }^{3}$. El "conflicto mapuche" y otras categorías y estereotipos asociados invierten las responsabilidades. Se presenta al indígena como culpable de su situación y ni la sociedad nacional ni el Estado asumen sus responsabilidades en una historia marcada por el colonialismo y racismo chileno (Saiz, Rapimán y Mladinic 2008; Saiz, Merino y Quilaqueo 2009; Richards 2016). La reproducción de prácticas coloniales en la investigación social hace necesaria la revisión crítica del corpus investigativo acerca de la cultura musical mapuche, sobre todo en lo que respecta a la etnografía, "arma de doble filo" en el estudio del "otro". Esperamos que la pesquisa de los trabajos etnográficos

1 Algunas reflexiones de este estudio forman parte de los proyectos FONDECYT N ${ }^{\circ} 11190505$ de la Agencia Nacional de Investigación y Desarrollo, y R05/17 de la Dirección de Investigación de la Universidad de Los Lagos. Asimismo, quisiéramos agradecer a Enrique Cámara y Jacob Rekedal por sus comentarios al borrador de nuestro artículo.

2 El etnomusicólogo Julio Mendívil (2016: 204-206) propone el uso plural del vocablo "música" para poner de manifiesto la diversidad de prácticas musicales. En este estudio hemos decidido incorporar la sugerencia del autor, por lo que a lo largo de nuestro artículo nos referiremos a las "músicas mapuche" con la intención de enfatizar la pluralidad de expresiones sonoras de este pueblo. Además, adherimos a la práctica generalizada en la actualidad de no pluralizar con la "s" castellana los términos en lengua mapuche.

${ }^{3}$ Saiz, Rapimán y Mladinic 2008, por mencionar algunos. 
relativos a las músicas de este pueblo originario nos permita reflexionar acerca de nuestras representaciones y procedimientos metodológicos, para así contribuir con la superación de esencialismos, visiones estereotipadas, apropiaciones y epistemologías coloniales.

Entendemos por etnografía al "proceso metodológico global que caracteriza a la antropología social, extendido luego al ámbito general de las ciencias sociales" (Velasco y Díaz de Rada 2004: 18). El trabajo de campo (de mediano o largo plazo) corresponde solo a una etapa de dicha metodología: el momento de observación y experimentación, en el que a su vez se utilizan diversas técnicas de recogida de "datos" (entrevistas, grabaciones de audio y de video, observación participante, por mencionar algunas). Otros momentos del proceso global pueden ser el análisis y la interpretación que el investigador o investigadora desarrolla en la tranquilidad de su escritorio, hasta finalizar en la redacción de un monográfico. Sin embargo, es bien sabido que esta supuesta secuencialidad es bastante ficticia. La etnografía se parece más a un proceso circular o en espiral que a uno lineal; es un ir y venir entre observaciones, vivencias, reflexiones, descripciones, traducciones, explicaciones, interpretaciones, representaciones, etc (Cámara 2004: 363).

Además, la etnografía también es comunicar; es un modo particular de escritura basada en narraciones y descripciones (Fassin 2016: 284-290). Es "vitalidad traducida en palabras" (Geertz 1989: 153). Si bien nuestra reflexión estará centrada en la etnografía como encuadre metodológico, algunas de las ideas que expondremos se referirán a la escritura etnográfica. Desde luego, somos conscientes de que el único modo de comunicar la experiencia de mujeres y hombres en la que los etnógrafos y etnógrafas nos introducimos no es la palabra, pero es el soporte que tendremos en cuenta en este artículo. Asimismo, somos conscientes de que las dos aproximaciones mencionadas, la etnografía en cuanto a proceso metodológico global y como estilo particular de escritura, no son los únicos modos de entenderla. Otros autores la comprenden como herramienta de la investigación cualitativa (Sisto 2008: 118). En definitiva, ya sea entendida como técnica, encuadre metodológico o tipo de escritura, deben reconocerse los múltiples sentidos y usos asociados a la etnografía en la actualidad, que hacen que hace décadas esta ya no sea patrimonio exclusivo de la antropología ni su empleo se restrinja al estudio de comunidades indígenas (Restrepo 2016: 15-34). Por todo lo anterior, hemos aclarado el modo en que entenderemos la etnografía en nuestro estudio: principalmente como proceso metodológico global y, secundariamente, como tipo de escritura; excluiremos en esta oportunidad su reducción a técnica de investigación.

Luego de la crisis de la representación, donde el problema ya no es solo la descripción de la diferencia cultural, sino también cómo se la representa (Clifford y Marcus 1986), y de las críticas poscoloniales, que apuntan al vínculo de disciplinas etnográficas -como la antropología- con el colonialismo (Said 2008 [1979], Spivak 2015 [1999]), la etnografía ha transitado de la recolección de datos al trabajo reflexivo y dialógico ${ }^{4}$. Si bien los enfoques tradicionales siguen reproduciéndose, muchos investigadores entienden el trabajo etnográfico como una actividad de construcción colectiva de saberes situados. Vicente Sisto aborda esta aproximación dialógica, desde la perspectiva de las ciencias sociales, y señala que uno de los elementos más significativos en este paradigma metodológico radica en el cambio "de la relación con el otro en el proceso de producción de la investigación" (Sisto 2008: 114). En este nuevo paradigma, la emergencia de diversas formas de etnografía a lo largo del último tiempo (etnografía performativa, autoetnografía, entrevistas activas reflexivas [Denzin 2001, Denzin y Lincoln 2005], etnografía virtual [Hine 2004], entre

4 Un texto clásico de ámbito norteamericano que intenta responder a las críticas poscoloniales a la etnografía y en el que se proponen y revisan nuevos modelos de trabajo de campo es Barz y Cooley 2008. 
otras) demandan una revisión profunda respecto de las formas en las que dicho método ha sido utilizado por la investigación musical.

Para el presente estudio hemos revisado investigaciones desarrolladas en Chile a partir de la década de 1970 hasta principios de 2019. No hemos considerado investigaciones acerca de comunidades mapuche de Argentina (o del Puelmapu) debido a que hemos preferido enfocarnos en trabajos desarrollados en el Gulumapu $u^{5}$, independiente de la procedencia del investigador o investigadora ${ }^{6}$. La determinación temporal de dichos estudios se justifica por cuanto durante esos años fueron publicados los primeros trabajos etnográficos relativos a música mapuche. Podrían considerarse los numerosos artículos de Carlos Isamitt, quien aplica "criterios etnomusicológicos que por esos años (la década de 1930-1940) lo sitúan en un nivel de gran avance metodológico" (Martínez 1996: 132). Sin embargo, dichos textos representan aproximaciones que, si bien son un importante aporte al estudio de las músicas mapuche, pertenecen a una etapa inicial en el estudio etnográfico del patrimonio musical de esas comunidades; Isamitt puede ser visto como un adelantado, ya que sus incursiones de largo aliento en terreno y sus reflexiones alejadas de los prejuicios de la época fueron desarrolladas durante décadas en las que la "etnografía profesional" se encontraba en pleno proceso de instalación en Norteamérica y Europa (Clifford 1995: 39-77). Posteriormente, las etnomusicólogas Isabel Aretz y María Ester Grebe son las primeras en realizar trabajo de campo en Chile bajo la influencia y en el marco de los planteamientos metodológicos y teóricos de las disciplinas etnográficas, en especial de la etnomusicología. Sus primeras publicaciones acerca de música mapuche coinciden precisamente con la década de $1970^{7}$.

Además del límite temporal y el lugar donde las investigaciones fueron desarrolladas (Gulumapu, en este caso), hemos considerado otros criterios para la selección de los textos revisados; estos son: pertinencia temática (que traten de músicas mapuche ${ }^{8}$ ) y que en sus estrategias metodológicas incluyan al menos algún "elemento" etnográfico. Se nos podrá objetar que varios de los textos que analizaremos no son ni pretenden ser etnografías. Efectivamente, así es. Si consideráramos solo etnografías con las premisas que hemos expuesto más arriba, el corpus que revisamos se vería considerablemente reducido. Por lo anterior, hemos decidido incluir trabajos que contienen algún "componente" etnográfico. Es decir, aquellos que utilizan técnicas (o herramientas) compartidas tanto por el encuadre metodológico que nos interesa como por otras metodologías de las ciencias sociales: la entrevista en sus distintas variantes (en profundidad, abierta, estructurada, semiestructurada) y el registro audiovisual en terreno. Asimismo, el uso de estas herramientas implica algún grado de incursión en el campo, por mínimo que este sea. Este es otro de los elementos que nos interesa en este artículo: dónde, cuándo y cómo se ha realizado trabajo de campo.

Si bien existen importantes trabajos históricos y arqueomusicológicos acerca de la cultura musical mapuche -algunos de los que reflexionan acerca del pasado etnográfico (Aracena 1997) y de los aportes y dificultades de la analogía etnográfica (Rondón y Vera

5 Denominación utilizada para referirse a las comunidades del lado oeste de la cordillera de los Andes, actual Chile. Las voces en mapudungun las señalamos con cursivas y utilizamos el Alfabeto Mapuche Unificado.

${ }^{6}$ La nacionalidad o filiación institucional de las autorías no constituyó un criterio de selecciónexclusión de los textos revisados, por lo que se han analizado trabajos de investigadoras e investigadores chilenos y de otros países.

7 Queremos ser claros en señalar que nos estamos refiriendo a las investigaciones acerca de música mapuche de comunidades localizadas en Chile. En esta misma década la etnomusicóloga Carol E. Robertson $(1976,1979)$ realizó etnografías con comunidades del Puelmapu (lado este de los Andes).

8 La adscripción disciplinaria de los autores y autoras de los textos revisados es diversa: va desde alguna de las ramas de la investigación musical a otras áreas de las ciencias sociales o humanidades. 
2008, Pérez de Arce 2007) ${ }^{9}$-, estos no se tendrán en cuenta en esta revisión, porque el interés del presente estudio es la etnografía en cuanto metodología que indaga prácticas y discursos musicales contemporáneos al investigador o investigadora; esto no implica que su perspectiva descarte la temporalidad o la historia ${ }^{10}$.

La literatura se ha rastreado mediante diversas fuentes de información: bibliotecas, repositorios de tesis, bases de datos académicas y espacios de difusión asociados a organizaciones mapuche, como por ejemplo la web mapuche.info ${ }^{11}$. En específico, el corpus de textos estudiados corresponde a treinta y dos trabajos académicos, publicados en español e inglés ${ }^{12}$, divididos entre tesis de posgrado (diez: Cayumil 2001, Martínez 1996, Painequeo 2000, Quilodrán 2013, Rekedal 2015, Silva-Zurita 2017, Soto-Silva 2018, Tapia 2007, Velásquez 2017, Villarroel 2012a), libros (tres: Avendaño, Millamán, Melillán y Brevis 2010; Caniguán y Villarroel 2011; Hernández 2003 [2001]), artículos científicos (diecisiete: Aretz 1970, Course 2009, González 1986, González y Oyarce 1986a, González y Oyarce 1986b, Grebe 1973, 1974, 1978, Martínez 2002, Painequeo 2012, Rekedal 2014a, 2014b, 2019, Rivas y Vidal 2014, Schindler 1997, Silva-Zurita 2014, Soto-Silva 2017), capítulos de libro (uno: Villarroel 2012b) y working papers (uno: Sepúlveda 2011). Hemos optado por estructurar nuestro análisis a partir de los siguientes aspectos metodológicos: temas de estudio, localización de estos, duración del trabajo de campo y técnicas de recolección de la información. Esta caracterización es seguida por una sección en la que comentamos los distintos modos en que la etnografía es entendida y utilizada -como metodología y texto- en las publicaciones revisadas.

\section{CARACTERIZACIÓN DE LOS ESTUDIOS REVISADOS}

Hemos utilizado la división propuesta por Javier Silva-Zurita (2017:104-110) para referirnos a las dos grandes áreas en las que es posible dividir los temas de estudio que emergen de nuestra revisión: aquellos dedicados al estudio de la música mapuche tradicional y los dedicados a la música mapuche no tradicional. La primera categoría, según el autor, consiste en aquella música practicada indistintamente en el contexto tradicional y no tradicional y estaría constituida por dos subcampos: la música ritual (connotaciones religiosas) y el ayekan (no religiosas). Silva-Zurita propone tres rasgos compartidos por la música mapuche tradicional: (1) solo utiliza instrumentos mapuche, (2) el texto de los cantos es mayoritariamente en mapudungun y el español es utilizado como complemento y (3) puede identificarse el uso de determinado material tonal, patrones rítmicos y técnicas estilísticas distintivas. La música mapuche no tradicional estaría constituida por aquella que no presenta los tres rasgos mencionados, pero en la que pueden identificarse algunos elementos aislados. Asimismo, estas músicas suelen incorporar el uso de géneros musicales como el hiphop,

9 Si bien José Pérez de Arce (2007) en su libro Música mapuche hace referencia a algunas comunicaciones personales de colaboradores mapuche, sus observaciones están basadas mayoritariamente en la revisión bibliográfica exhaustiva y en su señero trabajo arqueomusicológico. Por lo anterior, no hemos considerado en nuestra revisión este texto fundamental para el estudio de la cultura musical de la sociedad del Wallmapu.

10 Nos hemos esforzado por localizar la mayor cantidad posible de publicaciones acerca de músicas mapuche con las características antes señaladas. Esperamos no ofender a nadie si pasamos por alto algún trabajo en nuestra revisión.

11 http:/ /www.mapuche.info/ [acceso: 17 de agosto de 2020].

12 Excluimos de nuestra revisión los trabajos etnográficos en alemán acerca de música tradicional mapuche. Véanse Böning 1978 y Müller 2008. 
reguetón, cumbia, rock, entre otros. Utilizar esta distinción nos permite superar la dicotomía urbano/rural, que, además de representar una visión reduccionista y poco dinámica de la sociedad mapuche, puede prestarse para confusiones. Por ejemplo, si bien Jorge Martínez (2002) desarrolló su investigación en un contexto urbano (Santiago), la música mapuche que utiliza en su reflexión es tradicional (música de un palin). Por otro lado, algunos trabajos abarcan ambas categorías (Rivas y Vidal 2014, Sepúlveda 2011).

Veintiséis de los treinta y dos textos revisados versan sobre música mapuche tradicional (como mencionamos anteriormente, dos de ellos se ocupan, al mismo tiempo, de la expresión no tradicional). Los trabajos centrados en el estudio de la música mapuche no tradicional o parcialmente dedicados a ella son minoritarios en comparación con los dedicados a la categoría música tradicional: solo ocho de las treinta publicaciones consideradas en esta revisión. Además de esta división genérica, es posible establecer subtemáticas. Así, cinco textos se enfocan en instrumentos musicales mapuche, nueve en el canto mapuche y cuatro en educación y música mapuche. Es clara la preferencia por el estudio de la música mapuche tradicional. Solo desde la primera década del presente siglo se presta atención a nuevos fenómenos sonoros en el Wallmapu. Sin embargo, y a pesar del interés por la música tradicional por sobre la no tradicional, varios espacios, circunstancias y prácticas musicales tradicionales han sido escasamente investigadas como, por ejemplo, la música del ngillatun ${ }^{13}$.

En relación con las locaciones, es posible observar una distribución regional centrada principalmente en La Araucanía. En menor medida podemos encontrar estudios en las regiones de Los Ríos, Los Lagos y la Región Metropolitana. Las localidades preferidas para estos fines son lago Budi (nueve) y Puerto Saavedra (cinco) en La Araucanía; Osorno (dos) y Puerto Montt (dos) en Los Lagos y la ciudad de Santiago en la Región Metropolitana (dos). Es necesario señalar que aproximadamente tres estudios no indican su localización y otros mencionan localidades con menor presencia dentro de las regiones ya expuestas.

Existe un predominio significativo de las investigaciones localizadas en La Araucanía. Esto puede estar relacionado con el hecho de que el imaginario investigativo suele localizar en esta región el epicentro de las prácticas musicales mapuche. Si bien se infiere que el predominio de las investigaciones localizadas en la Región de La Araucanía se condice con que esta es la zona de mayor presencia histórica del pueblo mapuche y principal escenario del mal llamado "conflicto" mapuche, esta distribución representa un punto de reflexión importante. Aquel aspecto puede llegar a instalarse como un elemento particularmente complejo, ya que sugiere procesos de homogeneización de las identidades tradicionales. Como se ha señalado en algunas de las investigaciones estudiadas (Soto-Silva 2017 y 2018), la sociedad mapuche posee diversas variantes culturales a propósito de su distribución territorial. Estas variaciones ejercen un impacto importante en aspectos fundamentales de la cultura, así como el idioma y otras costumbres. Lo anterior sugiere un peligro: la homogeneización territorial en los estudios podría inducir a la generación de un estereotipo de prácticas musicales que no se correlaciona con las identidades territoriales presentes en dicho pueblo originario. Es más, dentro de la zona predominante en los textos revisados, la Región de La Araucanía, observamos una tendencia hacia un área en particular: el eje Puerto Saavedra-Budi.

En cuanto a la extensión del trabajo de campo, hemos podido observar una mayor variedad en los estudios revisados. En este sentido, en muchos casos ha resultado complejo determinar el tiempo exacto que los investigadores dedicaron al trabajo de campo, ya que

13 Asimismo, Margarita Canio Llanquinao y Gabriel Pozo Menares (2014: 85) señalan la carencia de etnografías acerca del pillañtun. 
algunos estudios no lo señalan o lo indican de modo ambiguo. Comúnmente, se observan menciones relacionadas con los lapsos de tiempo en los cuales se realizó la investigación. Sin embargo, la información asociada a las estadías realizadas no es sistemática. Sin perjuicio de esto, la revisión ha permitido establecer la siguiente categorización del tiempo dedicado al trabajo de campo en los diversos textos revisados: ocho señalan la duración exacta, trece aproximada y once no la indican. Esto es particularmente relevante, ya que nos señala que un porcentaje menor de los estudios $(25 \%)$ reporta en su estructura el detalle respecto de la extensión del trabajo de campo. Se puede observar que la mayoría de las publicaciones informa una duración aproximada $(40,6 \%)$ y una cantidad no menor omite dicho dato $(34,3 \%)$. Si se profundiza en el caso de las investigaciones que reportan tiempos exactos, la extensión suele ser de dos, tres, siete y ocho meses. Es decir, que el tiempo del trabajo de campo en los ocho textos que lo indican explícitamente tiene por lo general una duración inferior a un año, a excepción de los estudios de dos autores que forman parte de tesis doctorales y trabajos postdoctorales con financiamiento extranjero.

Estos resultados nos llevan a reflexionar acerca de las razones por las que la duración del trabajo de campo no es expuesta de manera explícita en la mayoría de los casos y por qué el trabajo de campo se suele remitir por lo general a periodos de tiempo inferiores a un año. Lo primero, podría deberse a que dicha información no ha sido considerada un aspecto relevante por las autorías que no lo indican. Segundo, la extensión puede estar limitada, en alguna medida, por las condiciones laborales de los investigadores en las universidades y por los mecanismos de financiación. Respecto del segundo aspecto, podemos mencionar que una de las vías de financiamiento de los trabajos analizados, los Fondos de Cultura del Ministerio de las Artes, Culturas y Patrimonio, solamente permiten la realización de estudios de un año de duración (considerando todas las etapas de la investigación). Esta situación podría complicar el desarrollo de trabajos de mayor envergadura, mientras que los concursos FONDECYT, de la Agencia Nacional de Investigación y Desarrollo de Chile, son altamente competitivos y se encuentran diseñados para investigadores insertos en la academia. Este escenario, sumado al número relativamente reducido de investigadores que trabajan estas temáticas, podría explicar en parte por qué el trabajo de campo es desarrollado de manera acotada en la mayoría de los casos. En este sentido, resulta revelador que los estudios que consideraron trabajos de campo de largo plazo (dos años o incluso más) fueron financiados con fondos extranjeros. Esto nos hace suponer que a nivel nacional existen razones internas, relacionadas con las condiciones laborales o de financiamiento estatal; y también de estilo, es decir, parece ser que la mayoría de los autores y autoras no consideran la duración del trabajo de campo un aspecto relevante al momento de describir sus procesos de investigación. Sin perjuicio de lo anterior, comprendemos que una experiencia intensiva en terreno en un periodo acotado puede ser igualmente significativa que un trabajo de campo de mayor extensión.

En cuanto a las técnicas e instrumentos de recolección de datos, encontramos una mayor homogeneidad en los mecanismos. La estrategia más utilizada es la entrevista abierta, seguida por la observación participante y no participante. Menos empleados son el grupo focal, entrevistas semiestructuradas, con guion, o el registro audiovisual. Respecto de las herramientas menos usadas, observamos que algunos estudios aportan información general: señalan haber realizado registros de audio y video; presumiblemente dichas grabaciones se han realizado en el contexto de observaciones no participantes. Como aspecto a reflexionar, es interesante que una de las técnicas más utilizadas sea la entrevista. En ningún caso discutimos lo valioso de esta herramienta; sin embargo, es posible que el uso exclusivo de entrevistas disminuya la profundización en los contextos e impida constatar las tensiones y discrepancias entre lo que se dice (relato), lo que se hace (observación) y las representaciones relativas a cómo debería ser tal o cual práctica (dimensión normativa). 


\section{ETNOGRAFÍAS: METODOLOGÍA Y TEXTO}

Nos parece necesario recordar que las clasificaciones y reflexiones de este apartado se basan en las premisas acerca de la etnografía que hemos expuesto en párrafos anteriores: nuestro modo operativo de entenderla en el marco del presente artículo es como un proceso global, un encuadre metodológico, que incluye técnicas, trabajo de campo, momentos de observación, análisis, descripción y reflexión, además de estrategias textuales. Es decir, que no consideraremos etnografía solamente la aplicación de la observación participante o de entrevistas; no la entenderemos únicamente como una herramienta (o técnica) de la investigación social. Yaunque consideramos que tampoco es únicamente un género literario, reconocemos que la metodología en cuestión tiene formas de escritura bien características. Es desde esta visión de la etnografía que realizamos las distinciones y críticas que siguen.

Como adelantamos, algunos de los trabajos analizados no son ni pretenden ser etnografías. A pesar de esto, los hemos incluido en nuestra revisión, pues utilizan algunas técnicas empleadas por etnógrafos, desde luego no exclusivas de este tipo de investigación social. Nos referimos a los aportes de orientación sociológica como el de Fabiana Rivas y Libertad Vidal (2014) acerca del folclore patrimonial y emergente de La Araucanía o la investigación de Ernesto Sepúlveda (2011) de los procesos de hibridación en música mapuche actual en sus diversos estilos. Los autores utilizan la entrevista en profundidad, herramienta de uso frecuente en el trabajo etnográfico. El análisis de este tipo de investigaciones nos permite contrastar el tipo de conclusiones a las que se llega por medio de diversas metodologías cualitativas. Esta es otra razón por la que incluimos dichas publicaciones en el presente artículo. Ambas investigaciones se centran en expresiones musicales mapuche poco estudiadas en ese momento. Las reflexiones de sus autores en torno a la relación de los discursos de los músicos a quienes entrevistaron y categorías como identidad, resistencia o industria son, sin duda alguna, aportes novedosos al estudio de la música mapuche. Su trabajo se centra en el análisis de lo dicho por los músicos, por tanto, no considera la observación de las prácticas musicales de sus entrevistados.

Otros trabajos que no pretenden ser una etnografía pero que sí incluyen la observación de prácticas son los de Martínez (1996, 2002). Su análisis transdisciplinario de dos ceremonias mapuche (palin y choyke purrun) en contexto urbano (Santiago de Chile) considera, además de entrevistas y conversaciones, el estudio de prácticas y el registro audiovisual. El autor no desarrolla un texto etnográfico, sino más bien una reflexión teórica que dialoga con la etnomusicología (entre otras disciplinas), propone modelos originales de análisis y considera la observación de prácticas, aunque no sepamos qué tan prolongadas fueron, pues no lo indica. Su artículo de 2002 se ha transformado en una referencia obligada para todo estudioso de las músicas mapuche.

En otro grupo de trabajos hay cierto gesto etnográfico en lo relativo a la metodología, pero no en lo textual (Painequeo 2000, 2012; Soto-Silva 2017, 2018; Velásquez 2017; Cayumil 2001; Avendaño, Millamán, Melillán y Brevis 2010). Aunque las investigaciones de Juan Painequeo $(2000,2012)$ no concuerden con nuestra definición operativa de etnografía, ciertos aspectos pueden ser considerados como etnográficos. El mismo autor lo reconoce cuando admite en su tesis el intento por desarrollar un estudio "lingüístico etnográfico". Lo etnográfico en este caso lo constituye no tanto el hecho de que algunos de los ül que analiza fueron registrados en el terreno, sino por la descripción de situaciones autobiográficas al referirse al modo de aprendizaje del canto mapuche. Además, podemos identificar que el componente etnográfico en los trabajos de Ignacio Soto-Silva es principalmente metodológico. Este está dado por el uso de las siguientes técnicas de recolección de datos: entrevista abierta en profundidad, entrevista semiestructurada y grupo focal (Soto-Silva 
2017), así como en la generación de instancias de conversación más distendidas y no jerarquizadas (Soto-Silva 2018).

Otra investigación realizada en este marco es la tesis de Ramón Cayumil (2001), cuyo estudio cualitativo, además de incluir el enfoque etnográfico de modo parcial, considera el autorreflexivo. Esto significa que, además del empleo de entrevistas y de la observación participante, el autor incorpora su experiencia personal en la investigación que realiza acerca de prácticas musicales, tanto en el contexto comunitario como en el escolar, y de cómo estas generan o afectan procesos de concientización y objetivación de la cultura mapuche. José Velásquez (2017) reconoce que su tesis no es una etnografía, sino una investigación cualitativa con orientación etnográfica. A pesar de lo anterior, su extenso trabajo de campo le permitió desarrollar numerosas observaciones de los variados contextos (ceremoniales y cotidianos) donde la música mapuche se practica.

El estudio de Elisa Avendaño, Rosamel Millamán, Claudio Melillán y Guido Brevis (2010) consistió fundamentalmente en la realización de entrevistas (en terreno y, en su mayoría, con autoridades tradicionales) y de grabaciones audiovisuales. Es decir, los autores utilizaron herramientas que suelen emplearse en las etnografías, pero no desarrollaron una etnografía en los términos que hemos definido en este artículo. Sin embargo, es interesante la metodología colaborativa que desarrollan, la que está conformada por cuatro perspectivas: mapuche kimün, científica, social y audiovisual. Además, y como destacan los propios autores, algunos de los integrantes del equipo son hablantes de mapudungun, conocedores de su cultura y del protocolo. Esto les permitió lograr un acercamiento fluido a las comunidades y a sus colaboradores. Que algunos de los investigadores fueran insiders es notorio en la profundidad de las entrevistas y reflexiones.

Los trabajos que siguen están basados en estancias en terreno más prolongadas y claramente delimitadas, lo que nos lleva a afirmar que su gesto etnográfico es más pronunciado, pero no construyen un texto etnográfico (Caniguán y Villarroel 2011, Villarroel 2012a, 2012b, Quilodrán 2013, Hernández 2003 [2001], Tapia 2007). Es el caso de varias publicaciones relativas al canto mapuche, algunas de autoría individual y otras colectiva, que nacen de estadías de trabajo de campo comunes. Nos referimos a los trabajos de Natalia Caniguán y Francisca Villarroel (2011) y Villarroel (2012a, 2012b). Sin duda, los diálogos que las autoras logran con sus colaboradores (en su mayoría ülkantufe) en torno a la importancia del canto, procesos de transmisión y enseñanza de la cultura por medio del $u ̈ l$, fueron posibles gracias al tiempo invertido en el trabajo de campo. Igualmente valiosos son los numerosos testimonios de los propios ülkantufe contenidos en sus textos y los cantos recopilados en el terreno. Lo que no incluyen las investigadoras en su trabajo, y que sería interesante desde una perspectiva etnográfica, es el análisis de las -sin duda- innumerables situaciones que compartieron con los ülkantufe. Recordemos que, al menos para ciertos autores, en la etnografía la descripción y el análisis de la situación no es ilustrativa de afirmaciones generales acerca de los procesos o mecanismos que estudiamos, ni menos floreos literarios. La escena "es el material mismo a partir del cual se elabora el análisis" (Fassin 2016: 285).

Observamos un enfoque similar al de las investigadoras en Rodrigo Quilodrán (2013). Parte del trabajo de campo fue realizado en el marco del mismo proyecto en el que participaron Caniguán y Villarroel, y fue complementado por el autor con largas estancias con otras comunidades. Es indudable su uso metodológico de la etnografía: realizó entrevistas y observación participante (en algunos casos participó como ejecutante), pero el estilo de su texto tampoco constituye una etnografía. Sus decisiones textuales podrían estar relacionadas con su enfoque principalmente sociológico. No obstante, excelentes etnografías han sido escritas por sociólogos.

Otro trabajo "a medio camino" es el de Jaime Hernández (2003 [2001]), quien gracias a su etnografía en el lago Maihue nos entrega interesantes datos no solo acerca de 
los instrumentos musicales mapuche tradicionales empleados (características generales, construcción, interpretación), sino también de las peculiaridades del ngillatun (o lepun) en esa zona. Su trabajo es una de las pocas referencias de música tradicional williche existentes hasta ahora. En el escrito, el autor incorpora las impresiones del etnógrafo y sobre todo abundantes citas a las palabras de sus interlocutores. En cuanto a texto etnográfico no es aún una referencia, pero sin duda contiene elementos de este tipo de narración.

Finalmente, podemos mencionar la tesis de Diego Tapia (2007). Para el autor, la etnografía es un método en el que el investigador se encuentra con el grupo que estudia, experiencia que supone una transformación en el etnógrafo. En su etnografía el autor afirma haber utilizado tres métodos: (1) mirar desde fuera con distancia, (2) participación sin intervención y (3) participación activa ${ }^{14}$. Pese a sus numerosas menciones y reflexiones a la etnografía y a la posición del etnógrafo, Tapia elabora un texto de discutible estilo etnográfico y de difícil lectura.

Un primer grupo de etnografías, desde nuestro punto de vista, lo constituyen los trabajos de Isabel Aretz (1970), María Ester Grebe (1973, 1974, 1978), Ernesto González (1986) y González y Ana María Oyarce (1986a, 1986b). Estos autores definen claramente su marco metodológico etnográfico y presentan textos principalmente descriptivos ("clásicos", podríamos decir). Este conjunto de etnografías establecen las bases del estudio etnomusicológico de las músicas mapuche.

Así, llegamos a las etnografías contemporáneas. La tesis de Silva-Zurita (2017), acerca de los rasgos sonoro-musicales más importantes de la música tradicional mapuche, no solo emplea la observación participante, sino que además detalla varios subroles: observadorespectador, músico participante, participante regular y aprendiz de música mapuche. Además, realiza entrevistas y revisa material audiovisual. En su texto reflexiona junto con sus interlocutores, lo que le permite llegar a uno de sus principales hallazgos: la categoría wünül. Dicha categoría es explicada por el autor de la siguiente forma: “(...) aborda elementos del sonido como altura, melodía, textura, ritmo y técnicas interpretativas en la ejecución y la improvisación, entre otros componentes que otorgan a la música una sonoridad tradicional" (Silva-Zurita 2017: 176) ${ }^{15}$; además de describir, gracias a su observación y participación, de modo exhaustivo ceremonias poco "etnografiadas" (y escasamente estudiadas desde otros enfoques) como es el caso del wetripantu (138-174).

También incluimos aquí algunos de los trabajos de Jacob Rekedal. En el capítulo cuatro de su tesis doctoral (Rekedal 2015: 164-234), el etnomusicólogo desarrolla una "etnografía de lo particular", estrategia propuesta por la antropóloga Lila Abu-Lughod (1991) ${ }^{16}$. Rekedal utiliza esta aproximación en dicho capítulo, donde se mezclan descripciones de un Mingako Kultural y reflexiones en torno a las relaciones entre ritual, música popular, artes, movimiento mapuche y autonomía. El tipo de etnografía que el autor desarrolla

14 A lo largo de nuestro artículo hemos evitado el uso de la categoría "método", debido a que el término puede involucrar discusiones que escapan a nuestros objetivos. En nuestro comentario al trabajo de Tapia hemos respetado el empleo del concepto señalado, ya que es el que el autor utiliza para referirse a la etnografía.

15 (...) addresses sound elements such as pitch, melody, texture, rhythm, interpretative techniques in performance and improvisatory practices, among other components that make a given music to sound traditional.

${ }^{16}$ La autora crítica una -tal vez la más frecuente-de las formas de operación y estilo de la escritura de las ciencias sociales: las generalizaciones. Estas, según Abu-Lughod, reafirman las jerarquías (es un lenguaje de poder), atenúan y homogenizan las diferencias y suprimen los conflictos y el tiempo. Por lo anterior, la autora propone escribir contra el concepto de cultura, categoría generalizadora que no escapa a la tendencia del esencialismo. Una de las estrategias sugeridas por Abu-Lughod para "escribir contra la cultura" son las "etnografías de lo particular". 
fusiona lo descriptivo con lo reflexivo. Si bien los protagonistas son sus interlocutores, en su aproximación la voz del etnógrafo no desaparece. En un texto posterior, Rekedal (2019) combina nuevamente el análisis musical con descripción etnográfica.

Por último, es importante mencionar en este grupo el artículo del antropólogo Magnus Course (2009) acerca del ül. Basado en un trabajo de campo intensivo, con una aproximación que mezcla las perspectivas sociológica y semiótica y en las preguntas que emergen de la "antropología musical" de Anthony Seeger (1987), el autor elabora una sugestiva propuesta relativa a la conexión entre las canciones tradicionales y la constitución dialógica de persona mapuche (che).

La utilización de la etnografía en los trabajos revisados es desigual. Así, observamos varias situaciones al respecto: (1) trabajos que utilizan técnicas que suelen ser empleadas por esta metodología, pero que, desde luego, son compartidas por otros encuadres metodológicos de las ciencias sociales, (2) otros que la emplean parcialmente (investigaciones cualitativas con componentes o perspectiva etnográfica), (3) otros en los que el gesto etnográfico es mayor, pero no presentan -o no logran- un texto etnográfico y (4) otros que sí emplean la etnografía como metodología y texto (en algunos casos de un modo "clásico" y otros más reflexivos). Con esta categorización no pretendemos alegar a favor de la etnografía como la metodología más adecuada para el estudio de la música, ni de la escritura etnográfica como el modo más conveniente para representar una determinada realidad. Consideramos que el gesto etnográfico en combinación con otras estrategias metodológicas puede contribuir con la pluralización de las miradas acerca de la dimensión sonora del mundo mapuche. Por supuesto, nos parece que ciertos elementos del trabajo etnográfico intensivo pueden ser destacados, ya que resultan pertinentes y ventajosos en contextos como el mapuche. Una etnografía de mediana o larga duración exige una inmersión más o menos prolongada y un conocimiento acabado no solo del tema específico, sino también del contexto. Desde nuestro punto de vista, es esta experiencia situada con personas, territorios y sonidos la que hace de la etnografía una posibilidad privilegiada para sumergirse en las complejidades de las músicas mapuche y en sus relaciones con los fenómenos sociales y políticos, sin dejar de considerar, por supuesto, los vínculos con los procesos coloniales y de resistencia, y sin abandonar la reflexión en torno a las políticas de la representación.

\section{CONCLUSIONES}

Nuestro análisis de los textos rastreados nos ha permitido elaborar algunas conclusiones relacionadas con la caracterización de las investigaciones y el modo en que comprenden el trabajo etnográfico. Respecto de las áreas temáticas más estudiadas, el predominio por el interés en la música mapuche tradicional es evidente, aunque observamos temáticas emergentes, vinculadas a la música no tradicional y popular urbana. Además, hemos notado que el lugar geográfico con mayor cantidad de estudios es la Región de La Araucanía, y particularmente el eje Puerto Saavedra-lago Budi. Los textos revisados muestran, en general, poca información respecto de la duración del trabajo de campo, lo que nos permite inferir que en muchos casos dicha información no se considera relevante.

Asimismo, hemos observado que la extensión del trabajo de campo parece constituir un aspecto condicionante al momento de encarar un modelo investigativo de las características señaladas. En este sentido, consideramos que la difundida utilización de la entrevista abierta sin que sea complementada con la observación participante en todos los casos, y el hecho de que la mayoría de los textos correspondan a investigaciones de inspiración etnográfica, pero no etnografías -al menos desde el punto de vista que hemos definido-, se relacionan con los disminuidos tiempos utilizados para el trabajo de campo. Si bien un 
trabajo de campo extenso no asegura una etnografía significativa, vale preguntarse: las etnografías de largo aliento, en este contexto, ¿no se han considerado necesarias? o ¿no han sido posibles? Probablemente ambas explicaciones tengan cierta validez. En tal sentido, nos preocupa la asistemática inclusión de la información relacionada con la duración del trabajo de campo e intuimos que posiblemente no sea un aspecto relevante para los investigadores e investigadoras.

Consideramos que las posibilidades de financiación pueden explicar en parte las limitaciones en la duración del trabajo de campo y la relevancia de este al momento de comunicar sus resultados. Como discutimos anteriormente, existe una correspondencia entre los trabajos de campo de mayor duración y las fuentes de financiamiento. Acerca de esto, la estructura de los proyectos de investigación financiados por los Fondos de Cultura del Ministerio de las Artes, Culturas y Patrimonio - quienes apoyan proyectos de investigación de un año de duración-, FONDECYT -que financia hasta tres años-, en complemento con las condiciones laborales de los investigadores al interior de las universidades chilenas, podrían condicionar en alguna medida la duración de la experiencia en terreno. Esto último se reafirma por cuanto los trabajos de mayor duración corresponden principalmente a estudios financiados con fondos extranjeros o a tesis doctorales (que por su condición permiten espacios amplios de indagación en terreno ${ }^{17}$, pero que no aseguran necesariamente una práctica investigativa continua y profesionalizada).

Independiente de las razones, nos parece evidente que el empleo de la etnografía, en tanto encuadre metodológico y estilo de escritura, ha sido escaso para el caso de las músicas mapuche. Asimismo, consideramos que un trabajo de campo de mayor extensión y procesos etnográficos de largo aliento permiten no solo la "recolección" de una mayor cantidad de información, sino también una mayor cercanía y comprensión del contexto donde las expresiones musicales que estudiamos tienen lugar.

Uno de los aspectos relevantes que deseamos reportar tiene relación con la evolución de la producción científica. Es particularmente interesante constatar el aparente aumento de investigadoras e investigadores dedicados a la producción de conocimiento acerca de las músicas mapuche ${ }^{18}$. Esto es significativo, por cuanto reafirma el interés de la comunidad científica, pero también es un buen predictor que nos permite ver con optimismo el desarrollo de dicha línea de investigación, en tanto se observa un creciente interés por el uso de la etnografía o alguno de sus elementos metodológicos.

Los artículos estudiados fueron realizados en el transcurso de cinco décadas. Durante la década comprendida entre 1970 y 1979 se pueden observar cuatro estudios de dos autoras; en el caso de las décadas de 1980 y 1990, es posible identificar seis publicaciones en total asociadas a cinco autores. Es desde la década del 2000 en adelante cuando observamos un incremento sustancial en la cantidad de publicaciones respecto de la temática en cuestión, con seis estudios entre 2000 y 2009 y diecisiete entre 2010 y 2019. Asimismo, es positivo observar un incremento sostenido en la cantidad de autores, de seis en el periodo 2000-2009 a doce en la última década. Esto indica un interés creciente por el estudio de las músicas mapuche (tanto de investigadores mapuche como no mapuche). Y aunque solo

17 Es necesario señalar que Becas Chile permite un máximo de un año de trabajo en terreno para el caso de los programas de doctorado de cuatro años de duración, y de nueve meses para los programas de tres años.

18 En otro contexto, es relevante señalar que hemos identificado una asimetría de género en quienes figuran como primer autor o autora: nueve corresponden a investigadoras y veintitrés a investigadores. Esto da cuenta de una brecha que, si bien escapa a los objetivos del presente artículo, nos plantea desafíos significativos que podrían ser abordados en futuros estudios. 
una minoría de los estudios analizados realizados en las últimas décadas son etnografías exhaustivas, la mayoría de los trabajos cualitativos consideran componentes etnográficos en sus metodologías.

Por otra parte, es importante señalar que tanto las etnografías contemporáneas como los estudios cualitativos recientes se ocupan tanto de la esfera tradicional como de la no tradicional. Esto muestra una apertura temática necesaria e inevitable. Tal vez la mayor aportación de los trabajos analizados en su conjunto sea la de reflejar la diversidad musical mapuche poniendo atención a variadas expresiones sonoras desde distintas aproximaciones. Por ejemplo, el canto mapuche ha sido estudiado desde el enfoque oralista y desde una perspectiva sociológico-semiótica, cuestión que desde luego revela diversos aspectos de una misma expresión musical ${ }^{19}$. Algunos trabajos se han preocupado por las características sonoro-musicales de la música mapuche y otros han reflexionado acerca de su papel en la reafirmación de la identidad étnica y territorial. La heterogeneidad en los temas y en los enfoques han permitido superar dicotomías que poco aportan a la comprensión de la música mapuche en la actualidad, como es la oposición entre espacios rurales y urbanos o la idea de que la música mapuche se desarrolla preferentemente en el ámbito ritual, premisa que cada vez es más refutada. En definitiva, observamos una variedad temática y epistemológica, pero que no va acompañada necesariamente de una reflexión metodológica. Es esta carencia la que nos condujo a la realización del presente trabajo que espera aportar al debate en torno a las implicancias y aportes de las metodologías utilizadas en las investigaciones dedicadas al estudio de las músicas de la sociedad mapuche y de otros grupos localizados en Chile o en zonas vecinas.

Por último, consideramos que la discusión metodológica no puede evitar la pertinente reflexión acerca de los procesos propios de la situación colonial en la que las expresiones sonoras mapuche son creadas, ejecutadas, reproducidas, estudiadas, situadas y disputadas. Nos referimos a la dimensión ética (y en cierto sentido, también epistemológica) que toda metodología debe llevar aparejada, en este caso la etnografía, proceso que puede conducir al establecimiento de relaciones genuinas y comprometidas, pero que al mismo tiempo puede reproducir formas de extractivismo. Y debemos reconocer que, así como el lugar que la etnografía ha ocupado en el estudio de las músicas mapuche ha sido discreto, la reflexión de la musicología chilena (y, tal vez, regional) acerca de las relaciones entre la experiencia musical y el colonialismo interno, así como nuestro aporte a la descolonización, han sido igualmente escasos.

19 Otro trabajo reciente acerca del ül o ülkantun es el de Pozo, Canio y Velásquez (2019). Los autores abordan el canto tradicional como estrategia para recordar el pasado, particularmente los tiempos de ocupación y despojo territorial a finales del siglo XIX y principios del XX. Además de la revisión de fuentes primarias, los autores realizaron trabajo de campo entre comunidades de la zona cordillerana. El artículo fue publicado el segundo semestre de 2019, por lo que no pudimos considerarlo en nuestra revisión. 


\section{BIBLIOGRAFÍA}

Abu-Lughod, LiLA

1991 "Writing against culture". Recapturing anthropology: working in the present. Richard G. Fox (editor). Santa Fe: School of American Research Press.

Aracena, Beth K.

1997 "Viewing the Ethnomusicological Past: Jesuit Influences on Araucanian Music in Colonial Chile”, Latin American Music Review / Revista de Música Latinoamericana, XVIII/1, pp. 1-29. DOI: $10.2307 / 780323$.

ARETZ, ISABEL

1970 "Cantos araucanos de mujeres", Revista Venezolana de Folklore, 3, pp. 73-104.

Avendaño, Elisa, Rosamel Millaman, Claudio Melillan y Guido Brevis

2010 Aukinkoi Ni Vlkantun. Temuco: Consejo Nacional de la Cultura y las Artes.

Barros, María José

2009 "La(s) identidad(es) mapuche(s) desde la ciudad global en mapurbe venganza a raíz de David Aniñir", Revista Chilena de Literatura, 75, pp. 29-46. DOI: 10.4067/S0718-22952009000200002.

Barz, Gregory y Timothy J. CoOley

2008 Shadows in the Field: New Perspectives for Fieldwork in Ethnomusicology. New York: Oxford University Press.

BÖNING, EWALD F.

1978 "Das Kultrún, die Machi-Trommel der Mapuche", Anthropos, LXXIII/5-6, pp. 817-44.

CÁmARa, EnRique

2004 Etnomusicología. Madrid: ICCMU.

Caniguán, Natalia y Francisca Villarroel

2011 Muñkupe ülkantun. Que el canto llegue a todas partes. Santiago: Consejo Nacional de la Cultura y las Artes.

Canio Llanquinao, Margarita y Gabriel Pozo Menares

2014 "Regina y Juan Salva: primeras grabaciones de cantos mapuches en soporte cilindros de fonógrafo (1905 y 1907)", Revista Musical Chilena, LXVIII/222, pp. 70-80. DOI: 10.4067/ S0716-27902014000200005.

Cayumil Calfigueo, Ramón

2001 "La música mapuche: Una mirada a los procesos subyacentes que se generan a partir del uso y práctica cotidiana en su uso y práctica en diferentes contextos socioculturales". Tesis para la obtención del título de Magíster en Educación Intercultural Bilingüe, mención Planificación y gestión en EIB. Cochabamaba: Universidad Mayor de San Simón.

Clifford, James

1995 "Sobre la autoridad etnográfica". Dilemas de la cultura. James Clifford (autor). Barcelona: Editorial Gedisa.

Clifford, James y George E. Marcus

1986 Writing Culture: The Poetics and Politics of Ethnography. Berkeley y Los Angeles, California: University of California Press.

Course, Magnus

2009 "Why Mapuche Sing", Journal of the Royal Anthropological Institute, XV/2, pp. 295-313. DOI: $10.1111 /$ j.1467-9655.2009.01554.x

DENZIN, NORMAN

2001 "The Reflexive Interview and a Performative Social Science", Qualitative Research 1, pp. 23-46. DOI: $10.1177 / 146879410100100102$. 
Denzin, Norman e Yvonna Lincoln

2005 Manual de investigación cualitativa. Barcelona: Gedisa.

FASSIN, DidiER

2016 La fuerza del orden: una etnografía del accionar policial en las periferias urbanas. Buenos Aires: Siglo Veintiuno Editores.

GeERTZ, CLIFFord

1989 El antropólogo como autor. Barcelona: Paidós.

GonZÁlez GreENhill, ERNesto

1986 "Vigencias de Instrumentos Musicales Mapuches", Revista Musical Chilena, XL/166, pp. 4-52.

González Greenhill, Ernesto y Ana María Oyarce Pisani

1986a "El trompe mapuche: Nuevos usos para un antiguo instrumento mapuche", Revista Musical Chilena, XL/166, pp. 53-67.

1986b "Kallfülikan, un canto mapuche. Descripción etnográfica, análisis musical y sus correspondencias con el aspecto literario", Actas de Lengua y Literatura Mapuche, 2, pp. 245-263.

Grebe, María Ester

1973 "El kultrún mapuche: un microcosmo simbólico", Revista Musical Chilena, XXVII/123-124, pp. 3-42.

1974 "Presencia del dualismo en la cultura y música mapuche", Revista Musical Chilena, XXVIII/126127, pp. 47-49.

1978 "Relationships between Music Practice and Cultural Context: The Kultrún and its Symbolism", The World of Music, XX/3, pp. 84-106.

Hernández OJEDA, JAime

2003 [2001] La música mapuche-williche del lago Maihue. Valdivia: Ediciones el Kultrún.

Hine, Christine

2004 Etnografia virtual. Barcelona: UOC (Universitat Oberta de Catalunya).

INE, Instituto Nacional de Estadísticas

2018 Segunda entrega resultados definitivos censo 2017. Santiago: INE, Instituto Nacional de Estadísticas.

Martínez UlloA, JoRge

1996 "Hacia una musicología transcultural: el anclaje de la identidad musical tradicional en mapuches urbanos". Tesis para optar al grado de Magíster en Artes, mención Musicología. Santiago: Universidad de Chile.

2002 "La música indígena y la identidad: los espacios musicales de las comunidades de mapuche urbanos", Revista Musical Chilena, LVI/198, pp. 21-44. DOI: 10.4067/S0716-27902002019800002.

Mendívil, Julio

2016 En contra de la música: herramientas para pensar, comprendery vivir las músicas. Ciudad Autónoma de Buenos Aires: Gourmet Musical Ediciones.

MÜLler, PhilipP

2008 Die Melodischen Strukturen des Ülkantun. Zum Einfluss universeller Klangprinzipien auf das mündlich tradierte Tonsystem der Mapuche-Indianer. München: Herbert Utz Verlag.

PAinequeo Paillán, JuAN

2000 Oralidad en el canto mapuche. Tesis para optar al grado de Maestro en Lingüística Indoamericana. México: Instituto Nacional Indigenista.

2012 “Técnicas de composición en el ül (canto mapuche)”, Literatura y lingüistica, 26, pp. 205-228. DOI: $10.4067 /$ S0716-58112012000200013. 
Pérez de Arce, José

2007 Música mapuche. Santiago de Chile: Fondo Nacional de Fomento del Libro y la Lectura.

Pozo Menares, Gabriel, Margarita Canio Llanquinao y José Velásquez Arce

2019 "Memoria oral mapuche a través de cantos tradicionales ülkantun: recordando la época de ocupación (siglos XIX y XX)”, Resonancias, XXIII/45, pp. 61-89. DOI: 10.7764/res.2019.45.3.

QUilodrán Vega, Rodrigo

2013 "La construcción de discursos. Globalización y música local mapuche”. Tesis para optar al grado de Magíster en Artes, mención Musicología. Santiago: Universidad de Chile.

REKEDAL, JACOB

2014a "El hip-hop mapuche en las fronteras de la expresión y el activismo", Revista de Lenguas y Literaturas Indoamericanas, 16, pp. 7-30.

2014b "Hip-hop Mapuche on the Araucanian Frontera", Alter/nativas Latin American Cultural Studies Journal 2/Spring, pp. 1-35.

2015 "Warrior Spirit: From Invasion to Fusion Music in the Mapuche Territory of Southern Chile". Tesis doctoral. Riverside: University of California.

2019 "Martyrdom and Mapuche Metal: Defying Cultural and Territorial Reductions in Twenty-First-Century Wallmapu”, Ethnomusicology, LXIII/1, pp. 78-104. DOI: 10.5406/ ethnomusicology.63.1.0078.

RESTREPo, EduARDo

2016 Etnografía: alcances, técnicas y éticas. Bogotá: Envión Editores.

Richards, PATricia

2016 Racismo. El modelo chileno y el multiculturalismo neoliberal bajo la concertación 1990-2010. Santiago: Pehuén.

Rivas Monje, Fabiana y Libertad Vidal Yévenes

2014 "Folclore de la Región de La Araucanía: Relaciones en su vínculo con la industria cultural musical y la expresión de identidad regional”, FRONTERAS I/2, pp. 81-110.

Robertson, Carol E.

1976 "Tayil as Category and Communication among the Argentine Mapuche: A Methodological Suggestion", Yearbook of the International Folk Music Council, 8, pp. 35-52. DOI: 10.2307/767381.

1979 "'Pulling the Ancestors': Performance Practice and Praxis in Mapuche Ordering”, Ethnomusicology, XXIII/3, pp. 395-416. DOI: 10.2307/850912.

Rondón, Víctor y Alejandro Vera

2008 "A propósito de nuevos sonidos para nuevos reinos: Prescripciones y prácticas músico-rituales en el área surandina colonial”, Latin American Music Review / Revista de Música Latinoamericana, XXIX/2, pp. 190-231. DOI: 10.1353/lat.0.0015.

SAID, EDWARD

2008 [1979] Orientalismo. Barcelona: Random House Mondadori.

Saiz, José Luis, María Eugenia Rapimán y Antonio Mladinic

2008 "Estereotipos sobre los mapuches: Su reciente evolución”, Psykhe, 17, pp. 27-40. DOI: 10.4067/ S0718-22282008000200003.

Saiz, José Luis, María Merino y Daniel Quilaqueo

2009 "Meta-estereotipos sobre los indígenas mapuches de Chile", Interdisciplinaria, 26, pp. 23-48.

SCHINDLER, Helmut

1997 "Una canción mapuche de Carlos Painenao para el Año Nuevo", Anthropos, XCII/1-3, pp. 129-138.

SEEGER, ANTHONY

1987 Why Suyá Sing: A Musical Anthropology of an Amazonian People. Cambridge: University Press. 
Sepúlveda Montiel, Ernesto

2011 Música mapuche actual. Recuperación cultural, resistencia, identidad. Nuke Mapuförlaget, Working Paper Series 38.

Silva-Zurita, Javier

2014 "Music and Cultural Identity: an Ethnically-based Music Program in a Mapuche-Pewenche School”, Eras, XVI/1, pp. 97-114.

2017 "An Ethnomusicological Study about the Main Musical Traits and Concepts of Traditional Mapuche Music". Tesis doctoral. Monash University, Faculty of Arts.

Sisto, VicENTE

2008 "La investigación como una aventura de producción dialógica: La relación con el otro y los criterios de validación en la metodología cualitativa contemporánea”, Psicoperspectivas, 7, pp. 114-136.

Soto-Silva, IgnACio

2017 "Música como Actividad Sociopolítica. Discursos de resistencia en la música urbana mapuche williche", Revista Vórtex, V/3, pp. 1-19.

2018 "Percepciones respecto a la música popular urbana en territorio mapuche williche". Tesis doctoral. Valladolid: Universidad de Valladolid.

Spivak, Gayatri Chakravorty

2015 Crítica de la razón poscolonial. Hacia una historia del presente evanescente. Madrid: Editorial Akal.

Tapia Carmagnani, Diego

2007 "El newen la fuerza que mueve a la música lafkenche en el lago Budi". Tesis para optar al grado de Magíster en Artes, mención Musicología. Santiago: Universidad de Chile.

Velasco, Honorio, y Ángel Díaz de Rada

2004 La lógica de la investigación etnográfica. Un modelo de trabajo para etnógrafos de la escuela. Madrid: Editorial Trotta.

Velásquez Arce, José

2017 "Patrimonio musical mapuche, su presencia en la comunidad y en la escuela. Consideraciones culturales necesarias para la enseñanza en el aula de música”. Tesis doctoral. Barcelona: Universidad Autónoma de Barcelona.

Villarroel, Francisca

2012a "El canto mapuche en el territorio del lago Budi, como expresión y transmisión de una memoria y cultura". Tesis para optar al grado de Magíster en Antropología. Santiago: Universidad Academia de Humanismo Cristiano.

2012b "El ül”. Mapuche. Procesos, politicas y culturas en el Chile del bicentenario. José Bengoa (editor). Santiago: Catalonia. 